\title{
Digital disciplinary differences: An analysis of computer-mediated science and 'Mode 2' knowledge production
}

\author{
Gaston Heimeriks ${ }^{\mathrm{a}, *}$, Peter van den Besselaar ${ }^{\mathrm{b}}$, Koen Frenken ${ }^{\mathrm{c}}$ \\ a The Dutch Advisory Council for Science and Technology Policy (AWT), Den Haag, The Netherlands \\ b Netherlands Center for Science System Assessment, Rathenau Instituut, Royal Netherlands Academy of Arts and Sciences E Amsterdam School of \\ Communication Research, Universiteit van Amsterdam, The Netherlands \\ ${ }^{c}$ Urban and Regional Research Centre Utrecht, Utrecht University, The Netherlands
}

\section{A R T I C L E I N F O}

\section{Article history:}

Received 12 December 2006

Received in revised form 2 April 2008

Accepted 23 May 2008

Available online 22 July 2008

\begin{abstract}
A B S T R A C T
The use of computer-mediated communications in research is one of the major shifts in processes of scientific knowledge production. We ask the question whether there are distinct disciplinary online communication patterns. In particular, we hypothesize that Mode 2 sciences have a higher use of Internet applications and address a greater variety of audiences with a greater variety of research outputs than Mode 1 sciences. Mode 2 was introduced as a descriptive and diagnostic characterization of transitions in knowledge production that are characterized by a range of features such as interdisciplinarity, reflexivity, focus on the context of application, heterogeneous actors, and a wide variety of types of output. This new mode of knowledge production has supposedly evolved out of the disciplinary and academic context of traditional ways in which knowledge was produced. It involves different mechanisms of generating and of communicating knowledge, more actors who come from different disciplines, and different sites in which knowledge is being produced.

We analyze online communication patterns in eight scientific disciplines including four Mode 1 sciences (High Energy Physics, Astrophysics, Literature Studies, and Psychology) and four Mode 2 sciences (Genetics, Biotechnology, Computer Science, and Information Science). We collected data on several dimensions of online communications, which included the shared set of outlinks of the departments and the characteristics of the websites in terms of size and types of files. The results suggest that web-based communications play a role in obtaining informational and financial resources, the use and exchange of digital data, the dissemination of results to academic audiences, and the dissemination of (non-traditional) output. The Internet maintains the three Mode 2 aspects of knowledge production: the interaction with non-academic partners, the dissemination of non-traditional output (software tools, databases, etc.), and the use of digital data. However, these characteristics of Mode 2 can be traced in different web attributes in each field. There is no systematic relationship between the three Mode 2 elements and the web characteristics under study here across all fields. This questions the usefulness of the Mode 2 label and underlines the specificity of scientific disciplines.
\end{abstract}

(c) 2008 Elsevier B.V. All rights reserved.

\footnotetext{
* Corresponding author.

E-mail addresses: GHeimeriks@gmail.com (G. Heimeriks), p.vandenbesselaar@rathenau.nl (P. van den Besselaar), k.frenken@geo.uu.nl (K. Frenken).
}

\section{Introduction}

According to a growing number of scholars, the organization of scientific knowledge production and its role in society is going through a transition. Traditional categorizations of human enterprise around which the world is 
organized, such as "science", "economy", "art" and "politics", now appear to be in flux, eroded, and socially contested. Instead, scientific knowledge production is said to have become increasingly interrelated with social and economic objectives and to take place outside the institutional regime provided by the university system and public research organizations. These developments are to be understood against the background of more general transitions including the end of the Cold War, the changing role of the nation state with the rise of regional and transnational structures, and the challenge of sustainability and global poverty reduction. More generally, the development of more 'open' systems of scientific knowledge production ${ }^{1}$ coincided with the increase in complexity in society and its increased reliance on formal knowledge for dealing with complex problems. In particular, Western economies have become crucially dependent on human capital and hightech entrepreneurship to create new sectors as engines of employment creation and productivity growth.

Portraying the impact and importance of this development are the heterogeneous ideas and concepts that have been introduced on the transitions in knowledge production in the information society in concepts of "national systems of innovation" (Lundvall, 1988), "research systems in transition" (Cozzens et al., 1990), "network society" (Castells, 1996), "the post modern research system" (Rip and Van der Meulen, 1996), "regional systems of innovation" (Cooke et al., 1998), "Triple Helix" (Etzkowitz and Leydesdorff, 1997), and the "creative class" (Florida, 2002). These concepts are indicative of the flux, reorganization, and the enhanced role of knowledge in the economy and society.

Among the earliest and most influential diagnoses dealing with transformations in knowledge production has been the Mode 2 concept (Gibbons et al., 1994). In this paper, it provided a central metaphor to start exploring the possibilities of mapping processes of knowledge production. Mode 2 was introduced as a descriptive and diagnostic characterization of transitions in knowledge production that are identified through a range of features like interdisciplinarity, reflexivity, focus on the context of application, heterogeneous actors, and a wide variety of types of output. According to Gibbons et al., this new mode of knowledge production has evolved out of the disciplinary and academic context of traditional ways in which knowledge was produced. Furthermore, the new mode involves different mechanisms of generating knowledge and of communicating them, more actors who come from different disciplines, and different sites in which knowledge is being produced (Gibbons et al., 1994; Nowotny et al., 2001).

The transitions in the processes of knowledge production described above coincide with the growth and development of information and communication technologies (ICTs) and their wide diffusion and application. Addressing these developments, Castells (1996) identifies the emergence of ICTs as a cause and effect of innovation. The emergence of the information society co-evolves with social and economic processes that are increas-

\footnotetext{
1 And open innovation (Chesbrough, 2003).
}

ingly information (and knowledge) intensive, and therefore the production, distribution, and use of information (and knowledge) are more and more central to all aspects of society. According to Castells, our society can be defined as an "Information Society": a society that depends on information and ICTs for all its processes and functions, and where knowledge is the most valuable asset. Communication of knowledge in a global network of interactions is at the same time the condition for innovation and a product of these innovations. Although information exchange has always been central to scientific research, the emergence of digital information, online accessible databases, and computermediated communication ( $\mathrm{CMC}$ ) has enabled new patterns of communication and collaboration within the science system and between science and society at large. Consequently, the study of the networks in which information is communicated is an appropriate instrument to analyze these social phenomena.

At the same time, the Mode 2 diagnosis identifies the role of ICTs as a carrier of the observed changes (Gibbons et al., 1994). The explosion of information and communication systems, which now permeate the world of business and finance and that of research alike, were fundamental in the transformation of knowledge institutions. Most obviously, ICTs have provided the means of dissolving the existing systematic differentiation of society (Luhmann, 1996). These technologies have helped to undermine national and institutional boundaries; they have undermined established social hierarchies and enabled geographically dispersed and distributed organizations (Nowotny et al., 2001). The Mode 2 thesis states that the sheer range of possibilities for new forms and intensities of communication opened up the possibility for a wider spectrum of cooperation within the knowledge production system (Gibbons et al., 1994). Consequently, in order to test the value of the Mode 2 thesis, it is important to specify further the role of ICTs in knowledge production.

The "Mode 2" diagnosis is not uncontested. Weingart (1997), for example, argues that Mode 2 knowledge production is not new at all, claiming that we may be merely returning to a balance between the two modes that was exhibited in an earlier eras. According to Weingart, Mode 2 is the original format of science before its academic institutionalization in the nineteenth century. Where have these ideas, which treat the scientist as an isolated individual and science as separated from the interests of society, come from? According to Etzkowitz and Leydesdorff, Mode 2 represents the material base of science, how it actually operates. They claim that Mode 1 is primarily a construct built upon that base in order to justify autonomy for science (Etzkowitz and Leydesdorff, 2000). Thus, from a policy perspective, the question of preserving the fragile Mode 1 knowledge production systems may be as important as the question of how to promote Mode 2 knowledge production (Dosi et al., 2005).

Rip (2002) also provides some nuances to these observations. What is described as a new development of transdisciplinarity, distributed, and fluid Mode 2 knowledge production is more accurately conceptualized as a product of long-term socio-cognitive evolution, in which the temporal and local stabilization of various dimensions 
of knowledge production cease to function in the information society. According to Rip, it is important to understand how a Mode 1 could emerge at all and get a hold on the variety of knowledge production and institutions. Mode 2 knowledge production is not uniform. In fact, he suggests that some features of Mode 2 have always been the rule rather than the exception (Rip, 2000). During the period when the Mode 1 research system came into existence, fields like chemistry, pharmacy, and electronics were progressing as much outside the academe as within it and with a lot of 'triple helix' interactions 'avant la lettre'.

In our view, the questions regarding the nature of Mode 2 knowledge production have thus become empirical. However, the empirical support for the aforementioned diagnoses about the supposed recent change in scientific knowledge production is more suggestive and anecdotal than systematic and conclusive in nature. Can we find evidence supporting the claim that distinctive forms of knowledge production exist? Can these differences be meaningfully characterized as Mode 1 and Mode 2 sciences? Finally, is the now popular distinction between Mode 1 and Mode 2 scientific knowledge production meaningful when studying the increasing use of ICTs in scientific communication? These are the questions we address using data on the use of online technologies in eight different disciplines, including four supposed Mode 1 sciences (High Energy Physics, Astrophysics, Literature Studies, and Psychology) and four supposed Mode 2 sciences (Genetics, Biotechnology, Computer Science, and Information Science).

\section{Computer-mediated science and 'Mode 2' knowledge production}

Our starting point is the observation that the growth and development of ICTs and their wide diffusion and application coincided with new modes of knowledge production (OECD, 1998). The thesis of Gibbons et al. (1994) claims that there is a new mode of knowledge production emerging in contrast to the more traditional Mode 1 of knowledge production. Mode 2 sciences can be described as interdisciplinary knowledge production with an emphasis on technological, social, and economic applications, subject to policy involvement and heterogeneity in producers and users of knowledge. Moreover, the types of output in Mode 2 knowledge production are expected to be more diverse than in traditional sciences.

According to the Mode 2 diagnosis, the local discovery environment of researchers is radically transformed by the increasing availability and use of ICTs. The online data repositories and Internet archives provide new ways of doing research. These new researching practices focus increasingly on monitoring, modeling, and mapping (De Jong and Rip, 1997). Furthermore, contemporary research focuses more on the properties and behavior of artifacts (such as computers, models, and information) rather than on natural phenomena in the real world (Gibbons et al., 1994). These observations are in agreement with the extensive use of data and models that Nentwich identified as having a positive effect on the use of ICTs in knowledge production (Nentwich, 2003).
In contrast to the more traditional form of knowledge production, the new mode of knowledge production counts not only scientific articles and books as output, but also tools, patents, technical norms, and social values. Nentwich (2003) found that a tradition of book publishing has a negative impact on the level of "cyberness", indicating a diverging use of media between Mode 1 and Mode 2 fields. This is supported by a case study of a Computer Science department, where we established that the Internet plays a prominent role in the dissemination of non-traditional output, such as software tools (Heimeriks, 2005). As the users of this 'new' output are to a large extent non-academic, this shows that what counts as relevant audiences in knowledge production is changing. Apart from the scientific quality, the economic potential and social implications of knowledge also play an important role in the system of Mode 2 sciences.

Following Gibbons et al. (1994), an increasing number of researchers and policy makers claim that the diffusion of ICTs and the emergence of Mode 2 knowledge production did not only coincide historically, but are also mutually reinforcing in their further development. Because of the ICT revolution, new patterns of communication and collaboration are expected to emerge in knowledge production, which are especially suited to support Mode 2 research activities (e.g., Castells, 2004 on biotechnology). The wide variety of data analysis tools, data storage, processing capacity, software tools, information delivery technologies, and electronic networks create a collaborative plurality and communicative heterogeneity. This claim needs still to be validated systematically by empirical research. There is evidence that disciplinary differences play an important role in explaining different patterns in ICT use. The size of academic disciplines, the possibilities of exploiting results, and the locus of critical information differ (Walsh and Bayma, 1996). For instance, only some disciplines moved substantial amounts of empirical data to online databases, notably, genetic sequences in genetics and digital images in astrophysics (OECD, 1998). Research costs, the necessity to collaborate, and the visibility of the work performed by scientists also vary across disciplines and can potentially influence the intensity of computer-mediated communication (Kling and McKim, 2000). There are also differences in communication conventions, including the increased pace of announcing new findings and the use of informal communication media. According to Kling and McKim (2000), the divide between fields where researchers share information freely ("open flow fields") and those where information flows are restricted is likely to change slowly, if at all. For example, unrestricted working article servers are used frequently in some areas of Physics, Computer Science, and a few Mathematical and Chemical subfields. But few biological or chemical specialties are expected to join forces with this venture, and to embrace it like High Energy Physics or Computer Science. However, it currently remains unclear whether the distinction between open flow fields and more closed sciences is transferable to the distinction between Mode 1 and Mode 2 sciences.

The increasing use of the web in knowledge production, not only as a means of communication and collaboration, but also as data and for dissemination of output of scientific 
fields, has become the focus of debate within science and science-policy circles (Heimeriks and Vasileiadou, 2008). The web is providing new means for the formal communication system based on peer-reviewed journals and face-to-face conferences (Vasileiadou, 2001), new fields such as webometrics treat online content and links as data which can provide information about the changing social environment and online repositories have pushed fields such as biomedicine in new directions (Lenoir, 1999). Aspects of this flux of ICTs and their increasing use in science and research have been termed cyberscience (Nentwich, 2003), e-science (Wouters, 2006), computermediated science (Heimeriks, 2005). These terms share the assumption that the use of ICTs conditions new types of research environments and have the potential of contributing to new ways of knowledge production.

Previous studies found that hyperlink networks between departments, compared to co-authorships and project cooperations, are much more diverse both in audiences that were addressed and in the communicated content (Heimeriks et al., 2003). This suggests that the web also serves as a social and public interface for research organizations. Concerning the differential use of Internet, higher Internet usage rates were found for natural sciences compared to social sciences and humanities (Abels et al., 1996), although more recent evidence suggest that social scientists have caught up (Walsh et al., 2000; Nentwich, 2003). Factors supporting the use of Internet in an academic discipline include a high importance on collaborative research, a cumulative tradition, and an extensive use of data and models. Furthermore, substantial external funding, an international orientation, and the role of visualization in research are also cited as important factors that positively influencing ICT use. In contrast to these, the affinity of a field to commercial exploitation, a local orientation, and its tradition of book publishing work against the use of Internet. However, after reviewing the evidence, Nentwich (2003) concluded that no single factor could explain the overall differences between the disciplines.

A potential methodological drawback of the studies mentioned is that, exceptions aside, results are based on the analysis of interview data only. Given the observed problems of using self-reporting methods to study online phenomena (Vasileiadou and Van den Besselaar, 2004), it would be useful to complement these qualitative studies with more quantitative data to explore these results further using the web. Another drawback of previous studies, pointed out by Barjak (2004), holds that these studies are often technology driven; they look at the use of Internet applications but fail to consider the functions for which the applications are needed. Additionally, the studies rarely put the use of computer-mediated networks into the general context of scientific communications. The present study attempts to overcome these limitations both methodologically and empirically.

In light of the previous studies, the following research questions did arise: 1 . Do we find empirical evidence for the existence of distinct online communication patterns across fields? 2. If so, what do these differences relate to? Are the web-based communications related to field specific use of the web, or can we identify more general patterns? In particular, is the distinction between 'Mode 1' and 'Mode 2' a useful one in understanding the use of the web?

Our main hypotheses are:

Hypothesis 1. Mode 2 sciences make more extensive use of Internet and Web applications.

Hypothesis 2. Mode 2 sciences are characterized by a greater variety of outputs disseminated through the web.

Hypothesis 3. Mode 2 sciences address a greater variety of audiences through the web.

In order to answer these questions, we mapped webbased communications and website characteristics of European academic departments in eight selected disciplines. We focused on several dimensions of online communications: the relationships that are established between organizations through hyperlinks and the size and content of the departmental websites. Furthermore, we attempted to establish a relationship between the "audiences" of the different fields and their online behavior.

\section{Data and methods}

\subsection{Data}

This study is based on web data concerning the size, content, and outlinks of the websites of universities and departments from $15^{2}$ EU member states (see Arroyo et al., 2003). Once web sites were identified and selected, some basic information was collected using software tools called 'mappers': the name of the department, the institution they belong to, and the URL that identifies them. These tools simply 'crawl' the web; they start from a certain site, follow the trace of its embedded links, and register the objects found during the process. The software program used to construct the database of European universities is Microsoft Site Analyst. ${ }^{3}$ All URLs were classified in three ways: an institutional code that classifies the type of entity based on a survey of the higher education systems in the European Union; a geographical code using the NUTS classification (Nomenclature of Territorial Units for Statistics) of EUROSTAT; and a thematic code according to the UNESCO classification of science and technology domains. The UNESCO codes have a three-level structure. The first two digits refer to the discipline, the third and fourth digits refer to fields, and the last two digits refer to subfields. In this study, the first four digits are used for the delineation of the fields.

\subsection{The cases}

The selected fields were: High Energy Physics, Astrophysics, Genetics, Biotechnology, Computer Science, Information Science, Literature Studies, and Psychology. The

\footnotetext{
2 This situation refers to the EU before the entrance of the 10 new member states in 2004.

3 This is a shareware version that was included by the manufacturer in the Back Office pack developed from software called Webmapper.
} 
data consists of website descriptions and outlinks that enable us to construct hyperlink networks between departments. The fields differ in terms of the relevant dimensions of knowledge production: we selected fields with more Mode 1 elements versus fields with more Mode 2 elements, and we included fields from the humanities, the social sciences, and the natural sciences. In our classification, Mode 2 refers to the emergence of a research system that is highly interactive and 'socially distributed' (Gibbons et al., 1994). Knowledge production used to be located primarily in scientific institutions and structured by scientific disciplines, however, its locations, practices, and principles are now much more heterogeneous. Mode 2 knowledge is produced 'in the context of application' by the so-called transdisciplinary collaborations. Moreover, scientists are more reflexive, and they operate according to non-traditional quality criteria when compared with the traditional disciplinary mode that relies on mechanisms such as peer review.

High Energy Physics (HEP) represents a clear example of a traditional Mode 1 field; research takes place in the traditional context of academia and research institutes and is organized along well-defined disciplinary identities. Globally, research in HEP is centered around a very small number of particle accelerators. Large-scale, distributed, global collaborations (Knorr Cetina, 1999; Traweek, 1988), and hyperauthorship (Cronin, 2001) are characteristics of this field. Major HEP projects are carried out by large, multiinstitutional collaborations, sometimes with budgets in the hundreds of millions of dollars. The primary equipment required for HEP experiments - colliding beam detectors - are extremely expensive, and therefore highly centralized in a few major laboratories. In the mid-1990s, there were only six colliding detectors in the world available to physicists (Galison, 1997). High-energy physicists have long led the sciences in their use of electronic media for sharing working papers. For example, most high-energy physicists contribute draft articles to an electronic working paper server at the time of submission of the article to a paper journal. While the paper journals are still important for archiving and for prestige and reward allocation, these electronic working paper servers are frequently the primary means of formal communication. The central role of the web in High Energy Physics is frequently attributed to the central role of high-energy physicists (most important, Tim Berners-Lee, from CERN) in having developed the basic protocols underlying the World Wide Web.

In addition to HEP, Astrophysics is often cited as an example of the enormous impact ICTs have on knowledge production and dissemination (OECD, 1998). Astrophysics can also be considered a typical example of the traditional Mode 1 type: disciplinary and academic knowledge production with a well-defined focus. Although Astrophysics and HEP are considered traditional academic disciplines in terms of their relevant audiences, they are also examples of government supported "big science". Knowledge production requires massive and unique machines like particle accelerators, which makes government funding inevitable (Price, 1963). Astrophysics and HEP both are characterized by a high importance on collaborative research, a cumulative tradition, substantial governmental funding, and an extensive use of data. According to Nentwich (2003), these are factors, which have a positive effect on the use of Internet tools in an academic discipline.

The Mode 2 field of Genetics is widely studied in relation to computer-mediated communication because of its use of large genome databases (as in the human genome project) that function as shared digital data-sources for genetics departments all over the world. The focus of much research is on the construction and manipulation of enormous amounts of digital data. In addition to universities, many companies and governments have been involved in these efforts. According to the OECD, the quick and successful achievements of the Human Genome Project would not have been possible in the absence of high-performance ICT (OECD, 1998). Genetics relies increasingly on simulations and modeling, and it operates in an increasingly interdisciplinary environment by combining experimental science with Information Sciences (Rip, 2002). The merging of digitization and genetics has also lead to new economic developments related to patented genome sequences. Consequently, there are cases where patents might still impede access to information. As Kling and McKim (2000) point out, in contrast with physicists, in Genetics, researchers typically circulate information only within small invisible colleges and broader access depends upon publication in journals. While a few electronic working paper servers have been established in a few biology subfields, such servers do not play a noticeable role in the communications system of Genetics. Many biological fields, however, do use digital corpora and other shared databases for important and growing data sets. Submission of gene sequences or other experimentally determined data into these shared databases is required before publication by many journals in Genetics and Molecular Biology. Published articles include "accession numbers" that identify datasets, which allow readers to obtain the research data almost instantly. Such digital corpora have become critical to the communication systems of research in life sciences. Interesting is that these biological databases operate synergistically with paper journals (Kling and McKim, 2000).

Computer Science (CS) is considered a typical example of a multidisciplinary Mode 2 field. CS is not characterized by a common orientation on data or theory, but by a focus on a variety of applications. The common skills that define the field are used for a variety of goals and topics, which are of interest to a wide variety of audiences and are not ordered into a common theoretical structure (Whitley, 2000). Previous research suggests that the type of output of CS departments (such as software tools) is often disseminated through the web to largely non-academic audiences (Heimeriks and Van den Besselaar, 2006). According to Kling (2000), Computer Science is an example of an open flow field where researchers share information freely. Barjak (2004) points out that researchers in Computer Science rely on the web as an important source of information, much more than is the case in other fields. Furthermore, in Computer Science, dependence on personal websites is more pronounced than in other disciplines when searching for information (Barjak, 2004).

Information Science (IS) is also considered a clear example of Mode 2 knowledge production, characterized by an 
interdisciplinary focus with emphasis on applications and a variety of producers and users of knowledge (Koehler, 2001). However, it belongs more to the social sciences and humanities, as it has its roots in the traditional field of library sciences. Information Science is expected to be greatly affected by the introduction of new ICTs (Nentwich, 2003). The availability of digital data, the increasing processing capacities, and the possibilities of disseminating information through the Internet are all factors that have a visible impact on Information Science. Research increasingly focuses on the use of online resources and on online information retrieval (Van den Besselaar and Heimeriks, 2006), which is expected to be reflected in the use of the Web in Information Science (Nentwich, 2003). Buckland describes how Information Science has been deeply influenced by technological developments in the form of new (online) standards, systems, and data (Buckland, 1999). Furthermore, the emergence of ICTs gave rise to a new kind of quantitative analysis within the field of Information Science, called "webometrics" (Van den Besselaar and Heimeriks, 2006). The web has also been the subject of information retrieval research by information scientists. Such studies have used web-link structures essentially on their own (Brin and Page, 1998) or in combination with textual analysis (Kleinberg, 1999). Many of these studies, as well as the data, are disseminated on the web. ${ }^{4}$

Biotechnology is another example of Mode 2 knowledge production characterized by an interdisciplinary focus with emphasis on applications, subject to policy involvement, and a variety of producers and users of knowledge (McKelvey, 1996). Moreover, Biotechnology is expected to have a strong commercial orientation. The combination of problem variety, instability, and multiple orderings of their importance with technical standardization occurs especially in this field (Whitley, 2000). However, it is unclear how ICTs have affected knowledge production in Biotechnology. The types of output and the use of data are not necessarily suited for web-based communications. Furthermore, Nentwich concludes that the affinity of a field to commercial exploitation works against the use of Web and Internet. Additionally, journal publications are considered very important in the field; however, E-journals have a very low prestige. Kling and McKim describe the biological sciences as characterized by a restricted information flow, a factor that has a negative impact on Internet use.

The field of the Literature Studies is used here as the paradigmatic example of a traditional Mode 1 field within the humanities. The Literature research is carried out in classical universities and is contextual and local in orientation. This implies that research skills are relatively diffuse and variable across knowledge objects and local cultures. Thus, local research groups tend to develop distinct ways of conducting and interpreting research on particular sorts of cognitive objects conceived in particular ways (Whitley, 2000). Scholars in the Literature Studies have a strong tradition in book publishing, a factor that Nentwich identified as having a negative impact on the level of 'cyberness'. As

\footnotetext{
${ }^{4}$ See for example the online journal Cybermetrics at: http://www. cindoc.csic.es/cybermetrics/.
}

pointed out, persistently lower Internet usage rates were found for scholars in humanities than for natural scientists (Abels et al., 1996). Furthermore, the humanities are expected to operate on a more local level than the social and natural sciences, resulting in a lower level of cyberness (Nentwich, 2003).

The dominant feature of the social and behavioral sciences is their intellectual variety and fluidity (Whitley, 2000). Recent evidence suggests that these fields have caught up in Internet usage rates with natural sciences. In social sciences, the Internet is used frequently as it provides increasingly essential information for research. Many official (governmental) organizations provide online access to documents and data (such as statistics), and there are a number of organizations engaged in collecting and distributing empirical research data (Nentwich, 2003). On the other hand, it has been claimed that within Psychology the use of information from the web is less than in other fields (Barjak, 2004). Furthermore, according to Whitley (2000), variations in strategies and methodologies across departments cannot be easily coordinated because they are neither precise nor formal enough to overcome communication problems. Consequently, we do not expect a coordinated and common use of the web in (Mode 1) fields like Psychology.

\subsection{Methodology}

The database we used contains a large set of departments, their website characteristics (such as size and types of files) and all outgoing hyperlinks from these departments, and their UNESCO field codes. Using this, we created lists of European departments in each of the selected fields. Table 1 shows the distribution of the departments over the 15 countries. The distribution over countries is reasonable (but we do not claim representativity).

Our study consisted of several steps. We will start in Section 4.1 exploring the relevant global context of the websites, which is operationalized here as the set of common outlinks of the departments in a field. As the number of outlinks is very large, we compiled a list of the 100 most frequently occurring organizations in the aggregated set of outlinks of the departments in each field. We only use unique outlinks of the departments. ${ }^{5}$ In this way, links represent (unvalued) relations between organizations. After having compiled the list with the top 100 linked organizations, we classify them into a variety of types: universities, governmental organizations, data repositories, and so on. The more the departments in a field link to same organizations, the more 'codified' a field is.

In Section 4.2, we map the national outlinks of a field, which indicate local resources and dissemination outlets. In this study, we use the Dutch departments in the various fields to provide us with an indication of the local context. This will also inform us about the value of the most

\footnotetext{
5 If a department has more than one link to another organization, this only counts as one. In other words, we count the number of departments that link to the same organization without taking into account whether a department has 100,10 or 1 link to that organization.
} 
Table 1

The distribution of the departments over the 15 EU countries

\begin{tabular}{|c|c|c|c|c|c|c|c|c|c|c|c|c|c|c|c|c|}
\hline Field & $\mathrm{AU}$ & $\mathrm{BE}$ & $\mathrm{DE}$ & DK & ES & FI & FR & GR & IE & IT & LU & NL & PT & SE & UK & Total \\
\hline CS & 68 & 41 & 383 & 14 & 205 & 70 & 108 & 17 & 39 & 55 & 0 & 95 & 26 & 34 & 334 & 1489 \\
\hline Astro & 3 & 1 & 2 & 0 & 7 & 1 & 1 & 0 & 1 & 1 & 0 & 4 & 4 & 1 & 15 & 41 \\
\hline Biotech & 3 & 3 & 19 & 5 & 17 & 4 & 10 & 1 & 1 & 4 & 0 & 9 & 1 & 5 & 21 & 103 \\
\hline Gen & 7 & 5 & 34 & 2 & 18 & 2 & 5 & 0 & 4 & 7 & 0 & 10 & 2 & 9 & 39 & 144 \\
\hline HEP & 0 & 2 & 3 & 0 & 1 & 1 & 0 & 1 & 0 & 1 & 0 & 6 & 0 & 0 & 1 & 16 \\
\hline IS & 11 & 8 & 43 & 6 & 32 & 8 & 11 & 3 & 4 & 21 & 1 & 7 & 4 & 7 & 51 & 217 \\
\hline Lit & 3 & 8 & 11 & 5 & 16 & 12 & 7 & 0 & 0 & 16 & 1 & 17 & 0 & 3 & 37 & 136 \\
\hline Psy & 1 & 3 & 14 & 0 & 16 & 1 & 0 & 0 & 2 & 3 & 0 & 6 & 0 & 0 & 7 & 53 \\
\hline Total & 96 & 71 & 509 & 32 & 312 & 99 & 142 & 22 & 51 & 108 & 2 & 154 & 37 & 59 & 505 & 2199 \\
\hline
\end{tabular}

Astro, Astrophysics; Biotech, Biotechnology; CS, Computer Science; Gen, Genetics; HEP, High Energy Physics; IS, Information Science; Lit, Literature Studies; Psy, Psychology.

common outlinks at the entire field level: if fields have a predominantly local orientation, the common set of linked websites at the (international) level may be of less value as an indicator of audiences and resources.

We continue in Section 5 with an analysis of the characteristics of the websites. This enables us to test the hypothesis about disciplinary differences in web use. This analysis is based on the outlinks and the content of the websites. The latter is defined in terms of the number and nature of the files the websites contain. We distinguish between outlinks and files per website and per webpage. We use data on the total number of pages of the website, the total number of outlinks, the number of images (gif, jpeg, etc.) on each website, the presence of web maps (wmp), the number of applications (java, docs, pdf, etc.), the number of audio files (wav, aiff, au, etc.), text files (rtf and txt), and video files (mpeg, etc.). The size and content of websites may indicate the importance and nature of web use in different fields.

Finally, in Section 6, we investigate whether a relation exists between the size and content of a website, and the number of inlinks a website receives from other departments in the field: the academic impact of websites. By doing so, we can indicate the nature of knowledge production, the context of application, and the importance of web-based communications. Furthermore, we can establish general patterns in web-based communications of scholarly departments.

\section{Variety in the disciplinary the outlink patterns}

\subsection{The hyperlink environments}

Do the various fields differ in terms of their outlink patterns? To answer this question, we first describe the shared elements in the outlink environment of the departments. This was done by compiling lists of the most frequently occurring websites in the outlinks of the departments within each field. The types of organizations that the fields link to are presented in Table 2. In each field, the 100 most important organizations are classified in the categories "university", "publishers and journals", "governmental organizations" (UN, European commission, Cordis, etc.), "companies" (Adobe, Microsoft, AOL, etc.), "professional organizations", "research organizations", "data repositories", and "archives". This classification informs us about the role of the web in knowledge production and dissemination.

Table 2 shows that in three of the Mode 1 fields (Astrophysics, Literature Studies, and Psychology) and in one Mode 2 field (Genetics), universities are the largest category in the outlink environment. In the other three Mode 2 fields (Computer Science, Information Science, and Biotechnology) and in one Mode 1 field (HEP), companies are the largest category. Differences exist in the frequency distributions, but it is clear that several organization types are visible in all fields: universities, companies, publishers, professional organizations, governmental organizations, and data repositories. It can therefore be argued that the Internet serves similar functions in all fields, albeit to rather different extents, as large differences in numbers are visible. In all fields, we find links to software companies and to Internet providers, but links to companies are most visible within Computer Science and Biotechnology. Publishers and journals are especially well represented in Genetics and Information Science and under-represented in Computer Science and - unexpectedly - in the Literature Studies. Governmental organizations are most occurring in Astrophysics, Information Science, and Biotechnology, while data-repositories are most important in Genetics. Archives only occur in Computer Science, Genetics, Astrophysics, and HEP. Data repositories occur in all fields, but in Genetics, they are a rather large category.

Not only do the link-environments differ, also the intensity of the hyperlink relations differs considerably between the fields. Table 3 lists the average number of links per department in each of the fields by category of organization. The intensity of hyperlink relations is largest in Astrophysics and HEP. If we look in the other direction, we find that link relations are the strongest with universities, companies, and publishers.

Up to here, we analyzed the nature of the hyperlink environments in terms of types of organizations. The next question is whether individual organizations are prominent in those hyperlink environments. In other words, do many departments in a field not only link to other departments or companies, but to a small number of specific departments, companies, or organizations? If this is the case, one may call the hyperlink 'codified': even if there is no general rule guiding link-behavior, many departments in a field 'automatically' link to a shared core set of organizations. In less codified fields, the departments do not share many 
Table 2

The distribution of different types of linked organizations

\begin{tabular}{|c|c|c|c|c|c|c|c|c|}
\hline \% Outlinks to (by field) & CS & Astro & Bio & Gen & HEP & IS & Lit & Psy \\
\hline Companies & 42 & 15 & 33 & 21 & 25 & 30 & 27 & 34 \\
\hline Publishers & 10 & 13 & 14 & 24 & 15 & 18 & 8 & 13 \\
\hline Universities & 29 & 36 & 29 & 28 & 23 & 28 & 46 & 43 \\
\hline Research organizations & 2 & 5 & 0 & 5 & 11 & 1 & 0 & 0 \\
\hline Professional organizations & 8 & 9 & 9 & 4 & 9 & 7 & 2 & 3 \\
\hline Governmental organizations & 4 & 16 & 13 & 3 & 7 & 13 & 10 & 4 \\
\hline Archives and data repositories & 3 & 4 & 2 & 13 & 5 & 3 & 4 & 3 \\
\hline Other & 2 & 2 & 0 & 2 & 5 & 0 & 3 & 0 \\
\hline
\end{tabular}

Cells: percentage of the links.

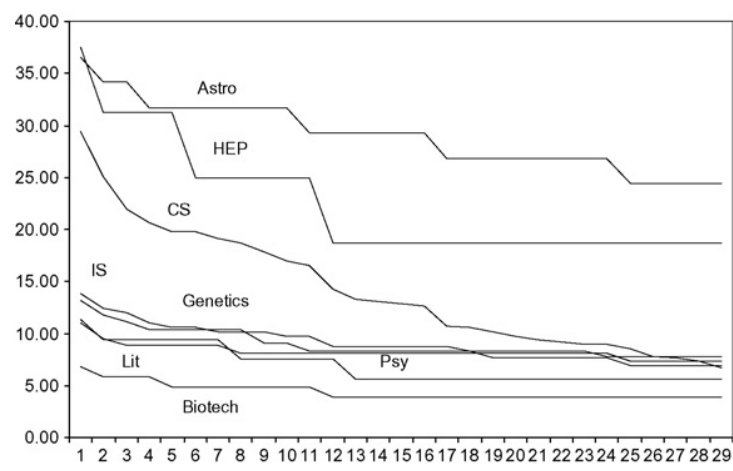

Fig. 1. The distribution of most frequently linked websites in selected fields.

outlinks. Despite the lack of clear mechanisms structuring online communications, a pattern of differences in codification seems to emerge in the fields under study (Fig. 1).

Fig. 1 shows the distribution of the share of departments in a field (on the $y$-axis) linking to the same organization (on the $x$-axis). The links to Internet-related companies such as Google, Yahoo, Microsoft, Amazon, and Adobe are excluded from the analyses in order to focus on field-specific outlinks only. Astrophysics shows the highest level of 'codification': 10 organizations in the environment of the field received links from more than $30 \%$ of the European departments in Astrophysics included in this study. On the other side of the spectrum, we find Information Science (IS), Literature Studies (Lit), Genetics, Psychology and Biotechnology. In these fields no organization exists that receives links from more than $15 \%$ of the departments.

What kind of websites receives many links? The level of 'codification' seems to depend on the use of shared data, on the importance of governmental and professional organizations and scientific publishers in the environment, and in some fields, on links to important university departments. More specifically, we find that links to peer departments are important within Computer Science, Astrophysics, and HEP. In the latter two, and in Genetics, global data repositories receive links from a large number of departments. In these fields, the most important global information repositories (NCBI, NASA, and CERN, respectively) clearly are the most frequently linked organizations. Shared links to companies are relatively important in Computer Science, in Information Science, and in Biotechnology. Publishers and journals are relatively important in Genetics. In addition to the NCBI data repository and the publisher Springer, the journals Nature and Science receive many links from the departments.

The analysis up to now is based on the top 100 most frequently occurring outlinks per research field, but the distribution of these most frequent outlinks over types of organizations may differ considerably from the less frequently occurring outlinks. Therefore, we randomly selected per research field some 100 unique outlinks, and the distribution of these outlinks over types of organization was compared with the distribution in the top 100 on field level. The table below lists the differences between the random sample and the top 100-again for each field. Table 4 shows the results and should be read as follows: in each cell, the number indicates how often a specific type of organization receives a link in the random group, compared to the top 100 group. For example, within Computer Science, $20 \%$ more links go to companies than in top 100 links (represented by the value 1.2 in the relevant cell). And the random set contains $40 \%$ less links to universities than the top 100 does (also for Computer Science-the relevant cell contains the value 0.6 )

The result show some general patterns in the outlinks of the samples compared to the most occurring (top 100) links on the field level. In the tail of the frequency distributions of all fields, we find more outlinks to companies and

Table 3

Average number of links per department by different types of organizations

\begin{tabular}{|c|c|c|c|c|c|c|c|c|}
\hline Links per department to & Comp & Astro & Bio & Gen & HEP & IS & Lit & Psy \\
\hline Companies & 4.6 & 3.6 & 1.5 & 1.5 & 5.0 & 2.6 & 2.4 & 2.6 \\
\hline Publishers & 1.0 & 3.5 & 0.7 & 1.9 & 3.3 & 1.7 & 0.6 & 0.8 \\
\hline Universities & 2.3 & 8.8 & 1.1 & 1.7 & 4.3 & 2.1 & 3.5 & 2.5 \\
\hline research organizations & 0.1 & 1.1 & 0.0 & 0.3 & 2.8 & 0.1 & 0.0 & 0.0 \\
\hline Professional organizations & 1.1 & 2.4 & 0.4 & 0.3 & 1.9 & 0.6 & 0.2 & 0.2 \\
\hline Governmental organizations & 0.5 & 4.0 & 0.6 & 0.2 & 1.5 & 1.0 & 0.9 & 0.4 \\
\hline Archives and Data repositories & 0.2 & 1.0 & 0.1 & 1.0 & 0.6 & 0.2 & 0.5 & 0.2 \\
\hline Average & 1.2 & 3.1 & 0.6 & 0.9 & 2.4 & 1.1 & 1.0 & 0.8 \\
\hline
\end{tabular}


Table 4

The outlink pattern of the random samples compared to the top-100

\begin{tabular}{|c|c|c|c|c|c|c|c|c|}
\hline Relative in random sample & Comp & Astro & Bio & Gen & HEP & IS & Lit & Psy \\
\hline Companies & 1.2 & 2.1 & 1.5 & 1.5 & 1.2 & 1.4 & 1.6 & 1.2 \\
\hline Publishers & 1.1 & 0.8 & 0.8 & 1.2 & 1.1 & 0.8 & 0.7 & 0.9 \\
\hline Universities & 0.6 & 0.8 & 0.7 & 0.6 & 0.9 & 0.8 & 0.8 & 0.8 \\
\hline Research organizations & 1.6 & 1.0 & 1.2 & 1.3 & 1.4 & 1.5 & 1.1 & 1.3 \\
\hline Professional organizations & 2.8 & 1.5 & - & 1.2 & 0.8 & 8.0 & - & - \\
\hline Governmental organizations & 0.5 & 0.6 & 0.5 & 1.0 & 0.7 & 0.2 & 1.8 & 2.0 \\
\hline Data repositories & 2.1 & 0.6 & 0.6 & 0.8 & 1.5 & 0.8 & 0.8 & 0.5 \\
\hline Archive & 1.0 & 0.4 & - & - & 0.6 & - & - & - \\
\hline
\end{tabular}

a Index: the top 100 links = 1.

to universities, but less links to publishers. Most fields show less links to professional organizations in the tail of the outlink distribution, except for the Literature Studies and Psychology. Possibly in these fields, the focus of research is more local in orientation, which may be reflected by the presence of local professional organizations in the outlink environment as local organization are expected to receive less links than global organizations. Consequently, one expects to find those in the tail of the distribution of outlinks. This stands in contrast to the international professional organizations that are generally found in the top 100 of linked organizations. The same pattern is found for data-repositories, which are also generally in the top 100 . These results also show us that in most fields, publishers and professional organizations are a defining element at the (international) field level.

\subsection{Outlinks: local or global?}

In order to further study what is not represented at the global field level - as indicated by the 100 most frequent outlinks - we also had a closer look at the local orientation of the departmental outlinks. The more a field is oriented towards a national and local context, the more it relies on local resources and dissemination outlets. In this study, we use the Dutch departments in the various fields to provide us with an indication of the local context. The percentage of the outlinks pointing to organizations in the same country differ between fields. Computer Science and Information Science have the lowest numbers of national outlinks (19\% and 20\%), and for Astrophysics and HEP the levels are 22\% and 26\%, respectively. Psychology and the Literature Studies are more locally oriented-28\% and $34 \%$ of the outlinks are within the country. Finally, in Biotechnology, 56\% of the departmental outlinks point to domestic organizations, which is much higher than in all other fields. In the related field of Genetics, the number of national outlinks stops at $28 \%{ }^{6}$

At the country level, the 'codification' of the departmental outlink structure is much higher than at the European field level. The most frequently occurring site per field ranges from $45 \%$ in the Literature Studies to $100 \%$ in Astrophysics and Information Science. Inspecting the lists of outlinks provides us with some explanations. In all fields,

\footnotetext{
6 These figures are for the Netherlands. In larger countries, national orientation may be larger: an organization in a large country simply has more local linking opportunities.
}

the most occurring links are predominantly domestic in origin. E.g., the 20 most occurring links in Dutch Biotechnology are all domestic. The lists also show that the web serves an important function in linking knowledge production with local audiences and resources. In addition to the National Organization for Scientific Research (NWO, the main Netherlands funding organization) and the Royal Netherlands Academy of Arts and Sciences (KNAW), local universities are well represented in all fields. In general, the types of organizations that are linked to by the Dutch departments, reflect a similar pattern as the tails of the outlink distribution at the field level discussed in the previous section.

\section{Differences in website characteristics}

After the exploration of the outlink environment of the fields in the previous sections, this section examines the characteristics of the websites of European departments in the selected fields. Since the target audiences and types of output vary across fields, we expect that the field differences in web use are not only reflected in the linking patterns of the departments' websites, but also in the size and content of the websites. We operationalize this in terms of the number of webpages, outlinks, images, videofiles, audio files, web-maps, applications, and total number of objects. These characteristics may vary between and within the different fields (Table 5), for example, there may be many images on the websites in one field but no or hardly any audio files. Using the SPSS routine ANOVA, we compared the website characteristics in the various fields. The analysis shows significant differences among the eight fields with respect to the number of pages, outlinks, images (gif, jpg, etc.), video-files (mpeg, etc.), and applications (java, docs, pdf, etc.) used by the departments in the various fields. No significant differences were found between fields for the number of gateways, audio files, web-maps, and the number of text files (txt and rtf).

Although the analysis produced significant differences for the set of eight fields, these differences are not always significant when comparing the fields pair-wise. In general, the closer the means of the distributions are between two fields, the less significant the differences are. Inspecting Table 5 shows this: the average website in Computer Science is relatively similar to the average Astrophysics website in many respects, and the average Biotechnology website resembles the average Genetics website. In general, the number of pages, outlinks, images, text files, and 
Table 5

List of site characteristics in selected fields (averages per site by field)

\begin{tabular}{|c|c|c|c|c|c|c|c|c|c|c|}
\hline \multicolumn{2}{|c|}{ Number of } & \multirow[t]{2}{*}{ Web pages } & \multirow[t]{2}{*}{ Out links } & \multirow[t]{2}{*}{ Images } & \multirow[t]{2}{*}{ Gate ways } & \multirow[t]{2}{*}{ Applications } & \multirow[t]{2}{*}{ Audio files } & \multirow[t]{2}{*}{ Video files } & \multirow[t]{2}{*}{ Text files } & \multirow[t]{2}{*}{ Web maps } \\
\hline Fields & $N$ & & & & & & & & & \\
\hline CS & 1489 & 1666 & 639 & 876 & 51 & 254 & 10 & 3.68 & 41 & 51 \\
\hline Astro & 41 & 1321 & 1073 & 1082 & 29 & 183 & 7.7 & 14 & 41 & 4.4 \\
\hline Bio & 103 & 413 & 147 & 371 & 10 & 47 & 0.2 & 0.7 & 0.4 & 0.2 \\
\hline Gen & 144 & 313 & 261 & 273 & 11 & 37 & 0.4 & 0.9 & 2 & 0.1 \\
\hline HEP & 16 & 519 & 445 & 1857 & 22 & 168 & 1.5 & 0.7 & 4.7 & 0.1 \\
\hline IS & 217 & 678 & 290 & 482 & 25 & 268 & 8.8 & 1.2 & 14 & 34 \\
\hline Lit & 136 & 350 & 279 & 220 & 18 & 27 & 4.2 & 0.2 & 0.8 & 0 \\
\hline Psy & 53 & 76 & 47 & 63 & 2 & 17 & 0.17 & 0 & 0.1 & 0 \\
\hline Anova & & .000 & .004 & .000 & .118 & .000 & .865 & .000 & .038 & .989 \\
\hline
\end{tabular}

applications on the websites provide the most significant distinctions between the fields.

The average size of the departmental sites show enormous differences (in the year of observation) ranging from 76 pages per site in the field of Psychology to 1665 pages per site in Computer Science. This suggests that in some fields departmental websites are a much less important medium for communication of data and output (Genetics, Biotechnology, Psychology, and Literature Studies). At the same time, Astrophysics and Computer Science research groups use their websites considerably. Located between those extremes are the fields of Information Science and HEP. ${ }^{7}$ Further research is needed to establish whether the size of the department (number of staff) correlates with the size of the web presence. Furthermore, it may be the case that research orientation versus teaching obligations plays a role when it comes to the departmental websites.

Not surprisingly, the highest average number of outlinks is found in large websites $(r=0.84)$. The size of the websites is related to the level of codification established in the previous section: the fields with large average websites show a more codified outlink pattern. The size of the website clearly indicates the importance of websites for communication and information dissemination. However, can we establish whether the size of the website is related to the presence of certain types of content (data or output) of the departments? We will now discuss the presence of different file types in more detail.

Very pronounced differences between the fields are visible in the number of images and video files that the websites contain. The websites in the fields of Astrophysics and High Energy Physics contain a large amount of digital visualizations, reflecting the role visual representation of numerical data has in those fields (OECD, 1998; Gooding, 2002). Moreover, it shows that web-based data sharing is important in these fields. More images are present in HEP, while many video files can be found in Astrophysics in addition to a relatively large number of images. Websites of departments in Computer Science also contain relatively large numbers of digital images and videos. This may be an indication of the important role computer vision related research plays. ${ }^{8}$

\footnotetext{
7 The differences in distribution between these groups are also significant in the pair-wise analyses.

8 This is not the case for audio files. Audio files were found to be present in all departmental websites with a distribution unrelated to the field.
}

Further evidence for the importance of the digital information stored on departmental websites is provided by the number of applications found on the websites of Astrophysics, High Energy Physics, Information Science, and Computer Science. The number of applications (java, docs, pdf, etc.) and the text files (txt, rtf) on the websites creates two groups of fields; Biotechnology, Genetics, Literature Studies, and Psychology have less than 47 application files on their websites on average. Computer Science, Astrophysics, High Energy Physics, and Information Science on the other hand contain -on average - more than 168 files per website. ${ }^{9}$ Additionally, the size of the websites here is decisive for the number of files and applications. In the cases of Computer Science, Astrophysics, and HEP, the importance of web-based communications was already established in relation to the role of digital data. Furthermore, the high number of applications in Computer Science and Information Science is easily understood, as the object of research in these fields increasingly is formed by digital information. Both fields are characterized by a strong focus on digital materials and applications.

Genetics has often been studied in relation to the importance of ICTs because of the crucial role large online databases play in this field. The very small average website size in the field of Genetics indicates that the role of online communications is not necessarily reflected in the size of the website. Here, the size of the website is a poor indicator for the importance of web-based communications. Above, we showed that the outlinks of the departments indicate the importance of central databases with genetic information. Relevant data are obviously not stored on the websites of the individual departments. Biotechnology, Literature Studies, and Psychology show little codification in the low number of shared outlinks, in addition to the characteristics of their websites, which indicate that digital data and digital output are generally not very important in these fields.

Above, we were discussing characteristics of websites. Table 6 summarizes the average webpage characteristics for each of the eight fields under study. The table indicates that the average size of the website is the main determining factor, as the variation is relatively low: characteristics per page are in the same order of magnitude for all the fields.

\footnotetext{
${ }^{9}$ These differences are significant between the two groups in the pairwise ANOVA's, but not significant between fields within the same group.
} 
Table 6

List of average page characteristics in selected fields

\begin{tabular}{|c|c|c|c|c|c|c|c|c|}
\hline Field & Out links & Images & Gate ways & Applications & Audio files & Video files & Text files & Web maps \\
\hline Comp & 0.38 & 0.53 & 0.03 & 0.15 & & & 0.02 & 0.03 \\
\hline Astro & 0.81 & 0.82 & 0.02 & 0.14 & 0.01 & 0.01 & 0.03 & \\
\hline Bio & 0.36 & 0.90 & 0.02 & 0.11 & & & & \\
\hline Gen & 0.83 & 0.87 & 0.04 & 0.12 & & & 0.01 & \\
\hline HEP & 0.86 & 3.58 & 0.04 & 0.32 & & & 0.01 & \\
\hline IS & 0.43 & 0.71 & 0.04 & 0.40 & 0.01 & & 0.02 & 0.05 \\
\hline Lit & 0.80 & 0.63 & 0.05 & 0.08 & 0.01 & & & \\
\hline $\begin{array}{l}\text { Psy } \\
\text { Coeffic }\end{array}$ & 0.62 & 0.83 & 0.03 & 0.22 & & & & \\
\hline
\end{tabular}

Empty cell: $<0.01$.

\section{Academic impact of the websites}

In the previous sections, we showed that the characteristics of the websites differ largely, as did the hyperlink patterns. In the analysis in this section, we investigate the relationship between the academic impact of a departmental website and the characteristics of its website. The academic impact (or importance) of a website is measured here in terms of the number of inlinks it receives from other departments in the field. The question to be answered is whether this academic web impact is based on (correlates with) characteristics of the website. We only use the important characteristics, such as numbers of pages, outlinks, images, and content (documents, databases, and so on). The analysis is done per research field, and the results are shown in Table 7.

All correlations are positive, indicating that in general large websites with a lot of content (documents, databases, spreadsheets, etc.) and outlinks are more popular and seem to have a larger academic impact than smaller sites with less outlinks and content. Inspecting the results in more detail, we do not find a systematic difference between the so-called Mode 1 and Mode 2 fields, nor between the fields with large, medium, and small size websites. The only 'systematic' difference seems to be between the Sciences, the Social Sciences, and Humanities: in the latter fields, the correlation between website characteristics and academic inlinks seems somewhat lower than in the sciences. Overall, the academic status of websites seems to be discipline specific - or even more department specific - and not much related to Mode 1 versus Mode 2 fields.

\section{Summary and discussion of the results}

In this paper we discussed the use of the web as a source of data to study the possible existence of distinct online communication patterns of European university depart-

Table 7

Correlation between academic inlinks and various website characteristics

\begin{tabular}{lllllllll}
\hline & Comp & Astro & Bio & Gen & HEP & IS & Lit & Psy \\
\hline Size & 0.47 & 0.67 & 0.78 & 0.61 & 0.40 & 0.20 & 0.63 & 0.20 \\
Outlinks & 0.34 & 0.43 & 0.66 & 0.51 & 0.29 & 0.46 & 0.31 & 0.46 \\
Images & 0.47 & 0.45 & 0.72 & 0.43 & 0.28 & 0.25 & 0.59 & 0.25 \\
Content & 0.51 & 0.81 & 0.45 & 0.44 & 0.69 & 0.35 & 0.44 & 0.35 \\
\hline
\end{tabular}

Italic: statistically not significant; size, number of pages; content, number of applications (text, data, programs). ments in eight different scientific fields. The results of our analyses established the existence of distinct online communication patterns in different fields. We found differences in terms of (a) the diversity of the outlink context; (b) the level of codification of outlinks; (c) the local orientation of the outlinks; (d) the size of the websites (statistically significant); (e) the number of outlinks (statistically significant), the number of images, applications, video files, and objects in the websites (statistically significant); and (f) we established a correlation of the website characteristics with the number of academic inlinks.

Although pronounced disciplinary differences are visible in the types of organizations in the hyperlinked context, it can be argued that there is a common element in the outlinks of all fields, mostly related to the traditional Mode 1 dimensions of knowledge production. Universities, publishers, and professional organizations play an important role in all fields, although to rather different extents. Additionally, the results showed that the web serves an important function in all fields, in linking knowledge production with local audiences and resources. We now summarize first the most salient findings per research fields. Then we reflect on the differences and similarities, and answer questions on whether the differences relate to differences between sciences and humanities, or between Mode 1 and Mode 2 research fields.

In Astrophysics, ICTs play an important role, as found in the website characteristics (for example, the exceptionally large number of video files used). The outlinks suggested a well-defined academic audience with a large set of shared outlinks, many to universities. Additionally, the high number of outlinks to governmental organizations indicates the role of government support. High Energy Physics departments link almost all to CERN and for the rest, to other academic institutions. In terms of content, the number of images on the websites is exceptionally high. In this discipline, the websites seem to be an important medium for communicating content to a predominant academic audience. Biotechnology - a clear example of Mode 2 knowledge production - has a focus on applications, is subject to policy involvement, and has heterogeneity in producers and users of knowledge. The websites are small and have little content, suggesting that the role of the web is small in this field. The (small number of) outlinks are local and have a strong commercial orientation. This latter orientation explains the low level of web use (Nentwich, 2003). In Genetics, websites are small, as is the number of outlinks. 
However, within the outlinks, those to international datarepositories have a prominent role, as expected. For the rest, outlinks seem domestically oriented, apart from links to publishers and scientific journals. This confirms that in Genetics, researchers typically circulate information only within smaller groups and broader access depends upon publication in journals (Kling and McKim, 2000). Here the distinction between fields with a restricted flow of information (like Biotechnology and Genetics) and fields with an open flow of information (like Astrophysics and High Energy Physics) becomes relevant and this relates of course to the economic potential of genetic and biotech data. Computer Science websites show a relatively high number of shared outlinks ('codification') and contain a large number of files and outlinks. The number of applications (content) is among the highest of the fields studied here. Furthermore, the outlinks have a more commercial orientation than other fields, suggesting the relevance of non-academic audiences in the field of Computer Science. Like in Computer Science, in Information Science, the web plays an important role, suggesting that the field has an 'open information flow'. Sites have more content (number of applications) than in any other field. The outlinks go to a variety of audiences (apart from other academic departments). A relatively large number of outlinks are directed to governmental organizations and companies, underlining a stronger application orientation than most other fields. Therefore, big websites are not necessarily full with academic output, and this explains the comparably low correlation of the number academic inlinks with website size and content. The websites of departments in the Literature Studies are generally very small and contain small numbers of files. The few and generally local oriented - outlinks indicate a mainly academic audience. In this paradigmatic example of a traditional Mode 1 field, scholars have a strong tradition in book publishing, a factor that Nentwich (2003) identified as having a negative impact on the level of 'cyberness'. Our analysis confirms this: in the hyperlink environment, we find many book publishers. Finally, Psychology represents a Mode 1 field in the social sciences. Websites are very small and maintain a small number of outlinks, showing that the web plays a minor role in the field (Barjak, 2004). Furthermore, there is little common orientation in the set of shared outlinks, and a large percentage of these outlinks were local.

The sample seems to group into two categories. In Astrophysics, High Energy Physics, Computer Science, and Information Science the web is used intensively, the number of shared outlinks is relatively high, the outlinks show an international orientation, and the number of webpages, outlinks, and content on the websites are high. A difference is that in the two physics specialties, data sharing is an important issue (NASA; CERN as the most linked organizations), whereas in the two other fields, it is not. And in Computer Science and Information Science, the relation with the non-academic environment seems stronger.

On the other hand, in Biotechnology, Genetics, Literature Studies, and Psychology, websites are on average small, have a modest content, and hardly share outlinks, which are more than often local. In some of the latter fields, this may indicate that the WWW is not very important yet, in others, such as Genetics, the size may be more a reflection of the restricted access to the data, rather than the data not being shared-as they are through NCBI.

In light of these results, we now turn to the three hypotheses formulated in the introduction about the relation between 'cyberscience' and changes in the knowledge production system:

(1) Mode 2 sciences make more extensive use of Internet applications than Mode 1 sciences.

(2) Mode 2 sciences disseminate a greater variety of outputs through the web compared to Mode 1 sciences.

(3) Mode 2 science address a greater variety of audiences through the web compared to Mode 1 sciences.

Firstly, the size of websites is obviously not related to the difference between Mode 2 and Mode 1 . Secondly, the same holds for the content of websites, in terms of applications, images, video and audio, and in numbers of outlinks. ${ }^{10}$ In other words, hypotheses 1 and 2 are not supported. If there is a relationship, we find it more between open information fields (like physics and computer/information science) and the fields with restricted information flows (like the life sciences). This relates more to the type of valorization of knowledge than to the question of whether application contexts play a role or not. The position of social sciences and humanities in this context needs further exploration. Another finding is that the early adopters of ICTs have the bigger sites (physics, computer/information science). The question is whether this is an issue of being behind (social sciences, humanities) or of variation, of heterogeneous developments.

Thirdly, outlink patterns were rather different, in terms of the codification, the type of linked organizations, and in terms of the shares of international links. Codification differed, and it was mainly related to the size of the websites and not to the Mode 2/Mode 1 distinction. The linked environments differed between the disciplines and could sometimes be related to specific Mode 2 characteristics of the field-but certainly not always. For example, disciplines like Computer Science, Biotechnology, and Information Science have many commercial outlinks, as one would expect given the economic role of these fields, but why this is also the case for High Energy Physics is less clear. Astrophysics and Literature had significantly more academic outlinks. On the other hand, it was not clear why one would expect more governmental outlinks in fields like Astrophysics, Biotechnology, Information Science, or Literature. Also the size of the outlink environment, its diversity, and its (inter)national orientation does differ, but is not related to 'Mode 2-ness'. In other words, outlink patterns were different between disciplines, but not systematically related to the Mode 1 versus Mode 2 distinction. In summary, hypothesis 3 was also not supported.

Finally, in all fields, we found that the size of sites (in terms of pages, content and outlinks) correlates relatively

\footnotetext{
10 If we compare the content per webpage between the fields, differences disappear-so in terms of building sites, a dominant design seems to emerge.
} 
strong with the academic impact of the site, but here also the strength of the correlations did not systematically differ between Mode 1 and Mode 2 fields.

\section{Conclusions}

As a general conclusion, the web does play an important role in facilitating the Mode 2 characteristics of knowledge production: in sharing data and information, in showing the network of the research organization, in supporting the interaction with non-academic partners, and in the dissemination of output. However, these characteristics of Mode 2 can be observed in each of the fields to a different extent. The distinction between Mode 1 and Mode 2 sciences therefore seems less a dichotomy. Rather, it is better to speak of Mode 1 and Mode 2 aspects of knowledge production, with each scientific field characterized by a mix of both characteristics. If such nuances are forgotten, terminologies quickly start to live a life on their own, and such lives tend to replicate extremely fast in academic and policy circles alike.

In line with Heimeriks and Vasileiadou (2008), we argue that Mode 2 characteristics are associated with the increasing use of information and communication technologies, but not in an identical way across all disciplines. The results show that the role of ICTs in knowledge production is different in different fields. In researching practices, ICTs provided innovations by facilitating the emergence of new maps, methods, models, and monitors. When it comes to exchanging results and building disciplinary identities, knowledge production may still be grounded in journal communications. However, additional information and reflexivity about information resources and databases may change the dynamics of these processes. Additionally, ICTs play an important role in enabling interactions between science and society in which socio-economical selection of "relevant" knowledge takes place. More specifically, in order to explain these observable reorganizations in knowledge production, we suggest an evolutionary conceptualization that relates these three aspects (Heimeriks and Vasileiadou, 2008). These dynamics do not necessarily translate into a single and stable configuration. Each of these dynamics may relate to the other two, which will result in the development of an emerging overlay of communications, networks, and organizations. Consequently, the resulting "search regime" is very field specific.

\section{Acknowledgements}

The work presented here was carried out at NIWI, Royal Netherlands Academy of Arts and Sciences. The paper is based on a revised version of chapter 7 of the $\mathrm{PhD}$ dissertation of Gaston Heimeriks. The research underlying this paper has been partially funded by the European Commission, grant IST-1999-20350, the EICSTES project. The project aimed at developing of web-based indicators for knowledge production, communication, and use. Partners are CINDOC (Spain), ARC Seibersdorf (Austria), DTI (Denmark), INIST/CNRS (France), NIWI-KNAW (Netherlands), and University of Surrey (UK). The authors would like to thank two anonymous reviewers, Eleftheria Vasileiadou and Arie Rip for useful comments on earlier drafts of this paper.

\section{References}

Abels, E.G., Liebscher, P., Denman, D.W., 1996. Factors that influence the use of electronic networks by science and engineering faculty at small institutions. Part I. Journal of the American Society for Information Science 47, 146-158.

Arroyo, N., Pareja, V.M., Aguillo, I., 2003. D3.2: Description of Web data in D3.1. CINDOC, Madrid. Available at: http://www.eicstes. org/EICSTES_PDF/Deliverables/Web\%20data\%20description.PDF.

Barjak, F., 2004. On the Integration of the Internet into Informal Science Communication. University of Applied Sciences Northwestern Switzerland, Solothurn.

Buckland, M., 1999. The landscape of information science: The American Society for Information Science at 62. Journal of the American Society for Information Science 50 (11), 970-974.

Castells, M., 1996. The Rise of the Network Society. Blackwell, Oxford.

Castells, M., 2004. Informationalism, networks, and the network society: a theoretical blueprint. In: Castells, M. (Ed.), The Network Society. A Cross-cultural Perspective. Edward Elgar, Cheltenham and Northampton, MA, pp. 3-48.

Chesbrough, H., 2003. Open Innovation: The New Imperative for Creating and Profiting from Technology. Harvard Business School Press, Cambridge.

Cooke, P., Uranga, M.G., Extebarria, G., 1998. Regional innovation systems: an evolutionary perspective. Environment and Planning A 30, 1563-1584.

Cozzens, S., Healey, P., Rip, A., Ziman, J. (Eds.), 1990. The Research System in Transition. Kluwer Academic Publishers, Boston, MA.

Cronin, B., 2001. Bibliometrics and beyond: some thoughts on Web-based citation analysis. Journal of Information Science 27 (1), 1-7.

De Jong, H., Rip, A., 1997. The computer revolution in science: steps towards the realization of computer-supported discovery environments. Artificial Intelligence 91, 225-256.

Dosi, G., Llerena, P., Sylos Labin, M., 2005. Science-technology-industry links and the "European Paradox": some notes on the dynamics of scientific and technological research in Europe. BETA Working Paper 2005-10. University of Strasbourg.

Etzkowitz, H., Leydesdorff, L., 2000. The dynamics of innovation: from national systems and "Mode 2" to a triple helix of university-industry-government relations. Introduction to the special 'Triple Helix' issue of Research Policy 29 (2), 109-123.

Florida, R., 2002. The Rise of the Creative Class. Basic Books, New York.

Galison, P., 1997. Image and Logic: A Material Culture of Physics. University of Chicago Press, Chicago, IL.

Gibbons, M.C., Limoges, H., Nowotny, S., Schwartzman, P.S., Trow, M., 1994. The New Production of Knowledge. Sage, London.

Gooding, D.C., 2002. Narrowing the cognitive span: experimentation, visualisation and digitalisation. In: Radder, H. (Ed.), Scientific Experimentation and its Philosophical Significance. University of Pittsburgh Press, Pittsburg.

Heimeriks, G.J., 2005. Knowledge production and communication in the information society. Mapping communications in heterogeneous research networks. PhD Dissertation. University of Amsterdam.

Heimeriks, G.J., Hörlesberger, M., Van den Besselaar, P., 2003. Mapping communication and collaboration in heterogeneous research networks. Scientometrics 58 (2), 391-413.

Heimeriks, G., Van den Besselaar, P., 2006. Analyzing hyperlinks networks; the meaning of hyperlink based indicators of knowledge production. Cybermetrics 10 (1).

Heimeriks, G., Vasileiadou, E., 2008. Changes or transition? Analysing the use of ICTs in sciences. Social Science Information 47 (1), 5-29.

Kling, R., McKim, G., 2000. Not just a matter of time: field differences and the shaping of electronic media in supporting scientific communication. Journal of the American Society for Information Science 51 (14), 1306-1320.

Knorr Cetina, K., 1999. Epistemic Cultures: How the Sciences make Knowledge. Harvard University Press.

Koehler, W., 2001. Information science as "Little Science”: The Implications of a bibliometric analysis of the Journal of the American Society for Information Science. Scientometrics 51 (1), 117-132.

Lenoir, T., 1999. Shaping biomedicine as an information science. In: Bowden, M.E., Hahn, T.B., Williams, R.V. (Eds.), Proceedings of the 1998 Conference on the History and Heritage of Science Information Systems. Information Today, Inc, Medford, NJ, pp. 27-45. 
Luhmann, N., 1996. Modern Society Shocked by Risks. Social Sciences Research Centre, Hong Kong, pp. 3-19.

Lundvall, B., 1988. Innovation as an interactive process: from user-producer interaction to the national system of innovation. In: Dosi, G., Freeman, C., Nelson, R., Silverberg, G., Soete, L. (Eds.), Technical Change and Economic Theory. Pinter, London, pp. 349-369.

McKelvey, M.D., 1996. Evolutionary Innovations: The Business of Biotechnology. Oxford University Press, Oxford.

Nentwich, M., 2003. Cyberscience, Research in the Age of the Internet. Austrian Academy of Sciences.

Nowotny, S., Scott, J., Gibbons, M., 2001. Re-Thinking Science. Knowledge and the Public in an Age of Uncertainty. Polity Press, Cambridge.

OECD, 1998. The Global Research Village: How Information and Communication Technologies Affect the Science System. OECD, Paris.

Price, D.d.S., 1963. Little Science, Big Science. Columbia University Press, New York.

Rip, A., 2000. Fashions, lock-ins, and the heterogeneity of knowledge production. In: Jacob, M., Hellström, T. (Eds.), The Future of Knowledge Production in the Academy. Open University Press, Buckingham.

Rip, A., 2002. Science for the 21st century. In: Tindemans, P., Verrijn-Stuart, A., Visser, R. (Eds.), The Future of the Sciences and Humanities. Amsterdam University Press, Amsterdam.

Rip, A., Van der Meulen, B., 1996. The post-modern research system. Science and Public Policy 23 (6), 343-352.
Traweek, S., 1988. Beamtimes and Lifetimes: The World of High Energy Physicists. Harvard University Press.

Van den Besselaar, P., Heimeriks, G.J., 2006. Mapping research topics using word-reference co-occurrences: a method and an exploratory case study. Scientometrics 68 (3).

Vasileiadou, E., 2001. Formal Scientific Communication through the Internet: The Case Study of Online Journals. University of Amsterdam, Amsterdam.

Vasileiadou, E., Van den Besselaar, P., 2004. One Method fits all? Studying a scientific emailing list combining different data and methods. In: New Research for New Media. Symposium "Innovative Research Methodologies", Terragona, Spain.

Walsh, J.P., Bayma, T., 1996. Computer networks and scientific work. Social Studies of Science 26, 661-703.

Walsh, J.P., Kucker, S., et al., 2000. Connecting minds: computer-mediated communication and scientific work. Journal of the American Society for Information Science 51 (14), 1295-1305.

Weingart, P., 1997. From "finalization" to "Mode 2": old wine in new bottles? Social Science Information 36 (4), 591-613.

Whitley, R., 2000. The Intellectual and Social Organization of the Sciences. Oxford University Press, Oxford.

Wouters, P., 2006. 'What is the matter with e-science: thinking aloud about informatization in knowledge creation', Pantaneto forum [available at: http://www.pantaneto.co.uk/issue23/wouters.htm]. 